\title{
Optofluidic channels in anti-resonant hollow-core fibers using focused ion beam
}

\author{
Wang, Yazhou; Adamu, Abubakar I.; Correa, Rodrigo Amezcua; Bang, Ole; Markos, Christos
}

\section{Published in:}

Proceedings of SPIE

Link to article, DOI:

$10.1117 / 12.2592409$

Publication date:

2021

Document Version

Publisher's PDF, also known as Version of record

Link back to DTU Orbit

Citation (APA):

Wang, Y., Adamu, A. I., Correa, R. A., Bang, O., \& Markos, C. (2021). Optofluidic channels in anti-resonant hollow-core fibers using focused ion beam. In K. Kalli, A. Mendez, \& P. Peterka (Eds.), Proceedings of SPIE (Vol. 11773). [1177315] SPIE - International Society for Optical Engineering. Proceedings of SPIE - The International Society for Optical Engineering Vol. 11773 https://doi.org/10.1117/12.2592409

\section{General rights}

Copyright and moral rights for the publications made accessible in the public portal are retained by the authors and/or other copyright owners and it is a condition of accessing publications that users recognise and abide by the legal requirements associated with these rights.

- Users may download and print one copy of any publication from the public portal for the purpose of private study or research.

- You may not further distribute the material or use it for any profit-making activity or commercial gain

- You may freely distribute the URL identifying the publication in the public portal 


\section{Optofluidic channels in anti-resonant hollow-core fibers using focused ion beam}

Wang, Yazhou, Adamu, Abubakar, Correa, Rodrigo, Bang, Ole, Markos, Christos

Yazhou Wang, Abubakar I. Adamu, Rodrigo Amezcua Correa, Ole Bang, Christos Markos, "Optofluidic channels in anti-resonant hollow-core fibers using focused ion beam," Proc. SPIE 11773, Micro-structured and Specialty Optical Fibres VII, 1177315 (18 April 2021); doi: 10.1117/12.2592409

SPIE. Event: SPIE Optics + Optoelectronics, 2021, Online Only 


\title{
Optofluidic channels in anti-resonant hollow-core fibers using focused ion beam
}

\author{
Yazhou Wang ${ }^{* a}$, Abubakar I. Adamu ${ }^{\mathrm{a}}$, Rodrigo Amezcua Correa ${ }^{\mathrm{b}}$, Ole Bang ${ }^{\mathrm{a}}$, Christos Markos ${ }^{\mathrm{a}}$ \\ ${ }^{a}$ DTU Fotonik, Technical University of Denmark, DK-2800 Kgs. Lyngby, Denmark. \\ ${ }^{b}$ CREOL, The College of Optics and Photonics, University of Central Florida, Orlando, FL-32816 \\ USA.
}

\begin{abstract}
Silica anti-resonant hollow-core fiber (ARHCF) is a promising platform for optofluidic applications because it can act as fluid-cell, permitting intense fluid-light interaction over extended length with low optical loss from ultra-violet to midinfrared region. For this kind of applications, an all-fiberized and compact structure is necessary. However, a prerequisite for this purpose is that micro-channels must be created on the side of the fiber, to provide access for the diffusion of fluids (i.e. liquid or gas) into the core. Several attempts based on femtosecond laser micro-machining technology have been made to create micro-channels through the silica cladding, but significant loss could be induced due to the damage of the cladding capillaries of ARHCF. Here, we report a high-precision and repeatable micro-machining technique using focused ion beam (FIB) milling on a nodeless ARHCF. $\mathrm{Ga}^{+}$ion beam is employed to bombard a $43 \mu \mathrm{m}$ thick outer cladding of ARHCF for 30 minutes, to create a $50 \mu \mathrm{m}$ deep fluidic channel. The micro-channel in the silica cladding is precisely drilled at the middle position of two adjacent capillaries with a $2.8 \mu \mathrm{m}$ gap, providing direct access for liquid/gas to diffuse into the hollow-core region, while avoiding the damage of the capillaries. Corroborating results from simulation of such a structure are presented to demonstrate that no additional loss is induced by the milled structure.
\end{abstract}

Keywords: Anti-resonant hollow-core fiber, ion beam, micro-channel, optofluidic application.

\section{INTRODUCTION}

Fluid-filled hollow-core fiber (HCF) technology has been playing an increasingly important role in optofluidic applications due to its advantages of high damage threshold, wide wavelength range, easy change of fluid medium, long light-fluid interaction distance, etc. ${ }^{1}$. The first low-loss HCF had a photonic bandgap (PBG) structure ${ }^{2}$, where the hollow core is surrounded by a periodic micro-structured cladding. The micro-structured cladding of a hollow core PBG is composed of holes that run through the bulk material (e.g. silica) and create a 2D crystal lattice that forbids certain frequencies from propagating through them. The light is therefore confined within the hollow core region during the propagates. The entire surrounding structure (cladding) is thus crucial for the guiding property of a hollow core PBG ${ }^{3}$. This is important to mention because many attempts have been made to create micro-channels through the holey-cladding, in order to enhance the diffusion of fluids into the fiber core region ${ }^{4-6}$. Micro-channels in HCFs are particularly necessary when such fibers are fusion-spliced to solid-core fibers ${ }^{7}$, to make compact and robust sensors, because splicing prevents fluid diffusion between the fiber core and external environment. Such diffusion, however, is essential for many applications in fluid-filled $5,8,9$ or liquid-filled ${ }^{10-12}$ HCF experiments, where interaction between fluid and light beam is required. The creation of access channels into the core that can survive splicing and does not increase the insertion loss of the fiber by light leaking out of the core, is thus of prime importance for the use of HCFs in sensing applications as well as towards development of compact gas-based fiber lasers using for the ultra-violet or mid-infrared spectral range ${ }^{13-15}$

The reported lowest loss induced by a micro-channel in an HCF is $\sim 0.35 \mathrm{~dB}$ for a single hole with a diameter of $\sim 500$ $\mathrm{nm}$, drilled with femtosecond laser ${ }^{4}$. In another report with a similar technique, a hole size of $20 \mu \mathrm{m}$ was shown to induce $\sim 0.5 \mathrm{~dB}$ loss per micro-channel in an HCF, under optimum conditions ${ }^{6}$. Other micro-fabrication techniques such as pressurized fusion splice method, results in an even higher loss of $6 \mathrm{~dB}$ for a single hole of $64 \mu \mathrm{m}$ wide ${ }^{16}$. All the aforementioned micro-channels are drilled in a PBG HCF, where loss induced by micro-defects in the fibers is almost inevitable due to the strong dependence of the guiding properties on the exact periodicity and symmetry of the PBG structure ${ }^{17}$. A relatively new type of HCF that has attracted significant interest in the HCF community is the anti-resonant HCF (ARHCF), because of its lower loss, single-modedness and broader transmission bandwidth when compared to hollow core PBG ${ }^{1,18}$. Furthermore, its simple structure significantly reduces fabrication complexity. In an ARHCF, light

Micro-structured and Specialty Optical Fibres VII, edited by Kyriacos Kalli, Alexis Mendez, Pavel Peterka, Proc. of SPIE Vol. 11773, 1177315 - (c) 2021 SPIE · CCC code: 0277-786X/21/\$21 · doi: 10.1117/12.2592409 
is guided in the fiber core region via the anti-resonant reflection optical waveguiding (ARROW) mechanism, which is achieved by surrounding the core region with a cladding made of single or multiple layers of capillary struts/tubes. The surrounding capillary tubes reflect light to the fiber core through ARROW mechanism, and they can either be touching ${ }^{19,20}$ or non-touching ${ }^{21,22}$. The later is also known as a nodeless tube lattice. Recently, an ARHCF was spliced to a standard step-index single-mode fiber (SMF), with a splice loss of $\sim 1.5 \mathrm{~dB}{ }^{23}$ despite the high mode-mismatch between the $40 \mu \mathrm{m}$ ARHCF and the $9 \mu \mathrm{m}$ SMF. A significantly lower splice loss of $0.3 \mathrm{~dB}$ has been demonstrated in PBG HCFs with mode field diameter similar to SMF ${ }^{24}$. Despite improvements in the splicing of these fibers, the pertinent problem that arises from blocking access to the core remains a challenge. Femtosecond laser ablation technology has been used to drill microchannel in ARHCF, inducing a relatively high loss of $\sim 1 \mathrm{~dB}$ per channel ${ }^{25}$ due to damages to the capillaries. A very recent study has used femtosecond laser micromachining of smooth channels in ARHCFs to access the core of the fiber, achieving a big channel size of $150 \mu \mathrm{m}$ with an induced loss of $0.45 \mathrm{~dB}^{26}$. This is still higher than the $0.35 \mathrm{~dB}$ loss reported in ${ }^{4}$ although with a much larger hole size. In these reports, due to the geometry of the fibers and the lack of real-time imaging during micromachining, damage to the capillary elements of the fiber is almost inevitable, consequently inducing additional loss.

In this paper, we present a novel approach of using a well-known ion beam milling technique to create a channel between the adjacent capillaries of a nodeless ARHCF, to provide access for fluid transfer to the fiber core, even when the ARHCF is spliced to a solid-core SMF. This technique takes advantage of the capillary separation in nodeless ARHCF and the fact that the outer cladding region is not involved in the ARROW mechanism. Consequently, high precision ion beam milling could be used to drill micro-channels on the outer cladding, while the gap between the non-touching capillaries of the ARHCF serves as a natural aisle between the core region and the micro-channels. This minimizes and even completely eliminates additional loss as a result of the micro-machining, which is corroborated by numerical simulations.

\section{SIMULATION OF MILLED STRUCTURE ON ARHCF}
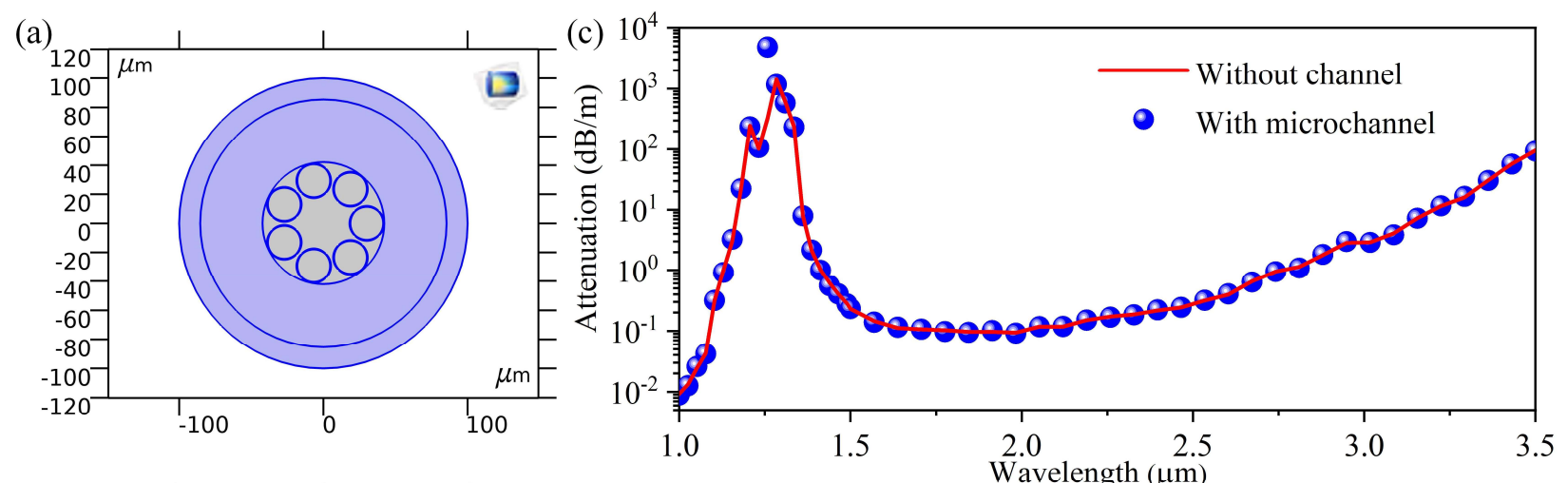

(b)
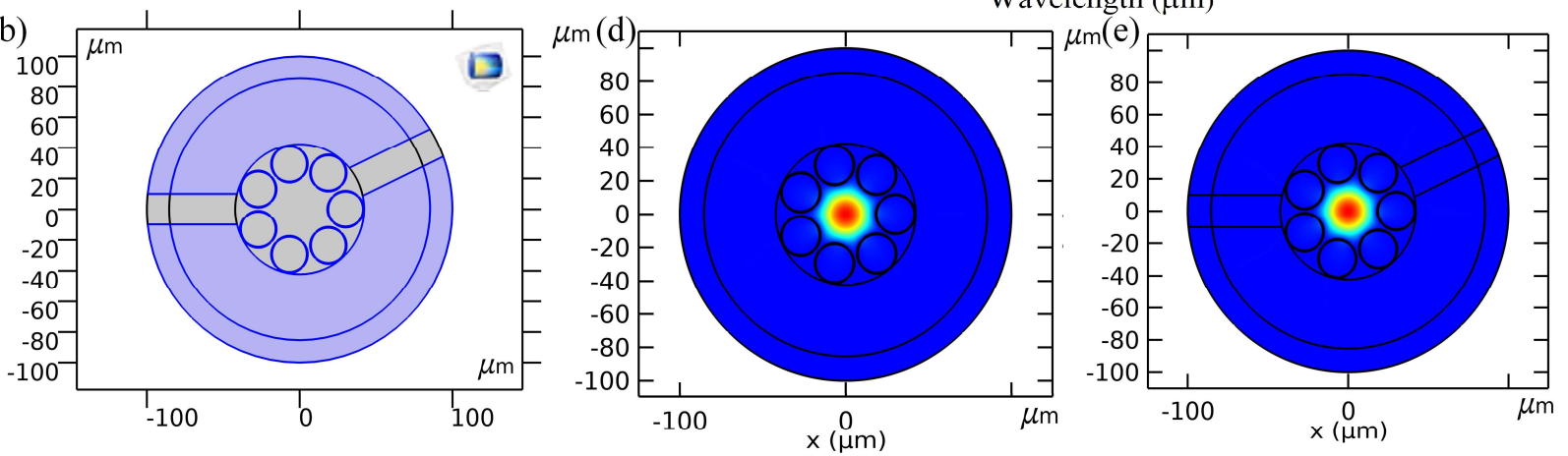

Figure 1. Design of ARHCF for simulation in COMSOL with and without micro-channels in (a) and (b), respectively. The light-blue part donates the silica region, and the grey part donates the air part. (c) Comparison between the simulated loss profiles. The red curve is without any micro-channel and the blue dot is with two micro-channels. (d) and (e) are simulated fundamental mode fields at $2.5 \mu \mathrm{m}$ without/with micro-channels, respectively. 
First, the finite-element method based on a commercial software (COMSOL) is adopted to demonstrate the impact of the micro-channel on the loss performance of the ARHCF. A micro-channel, positioned in the outer cladding between two non-touching capillaries, will allow access of fluids to the fiber core. Figure 1(a) shows the designed ARHCF in 2D space. It has a core size of $46 \mu \mathrm{m}$, surrounded by seven non-touching silica capillaries, with a wall thickness of $640 \mathrm{~nm}$. The minimum distance between adjacent capillaries is $2.83 \mu \mathrm{m}$. There is an outer cladding for holding all the silica capillaries, and the target of this work is to make optofluidic channels through it, as shown by Figure 1(b). During the simulation, a perfectly matched layer is used as the boundary condition. A fine mesh size ranging from $\lambda / 6$ to $\lambda / 4$ is adopted for all the geometry regions (includes air core, silica cladding, and PML), to ensure simulation accuracy. Surface scattering loss was ignored because the inner surface roughness of the waveguide is much smaller than the laser wavelength in the infrared region and also due to the large core diameter of the ARHCF ${ }^{21}$. The design in Fig. 1(c) with two channels in the outside silica cladding part indicates that the fiber in 3D space has two long slits of $30 \mu \mathrm{m} \times 43 \mu \mathrm{m}$ along its axial direction, which has a much larger core-exposed area than the experimental case where only two slits of $50 \mu \mathrm{m}$ depth.

The channels in Figure 1(b) indicates that the fiber in 3D space has two long slits of $30 \mu \mathrm{m} \times 43 \mu \mathrm{m}$ along its axial direction, which has a much larger core-exposed area than the experimental case where only two slits of $50 \mu \mathrm{m}$ depth. Even with such long slits, the simulation results in Figure 1(c) show that the fiber loss of machined fiber remains unchanged when compared to the fiber without channels. Both of them gradually increases towards the longer wavelength due to the increased material loss of silica and overlap with the fundamental mode at that wavelength. This result suggests that altering the outside silica cladding part has little to no impact on the optical performance of the anti-resonant fiber, which is consistent with the conclusion in Ref. ${ }^{27}$. As a result, the fundamental mode for the two cases have the same intensity distribution and diameter, as indicated by Figure 1(d) and 1(e).

\section{FABRICATION AND FIB MICROMACHINING}
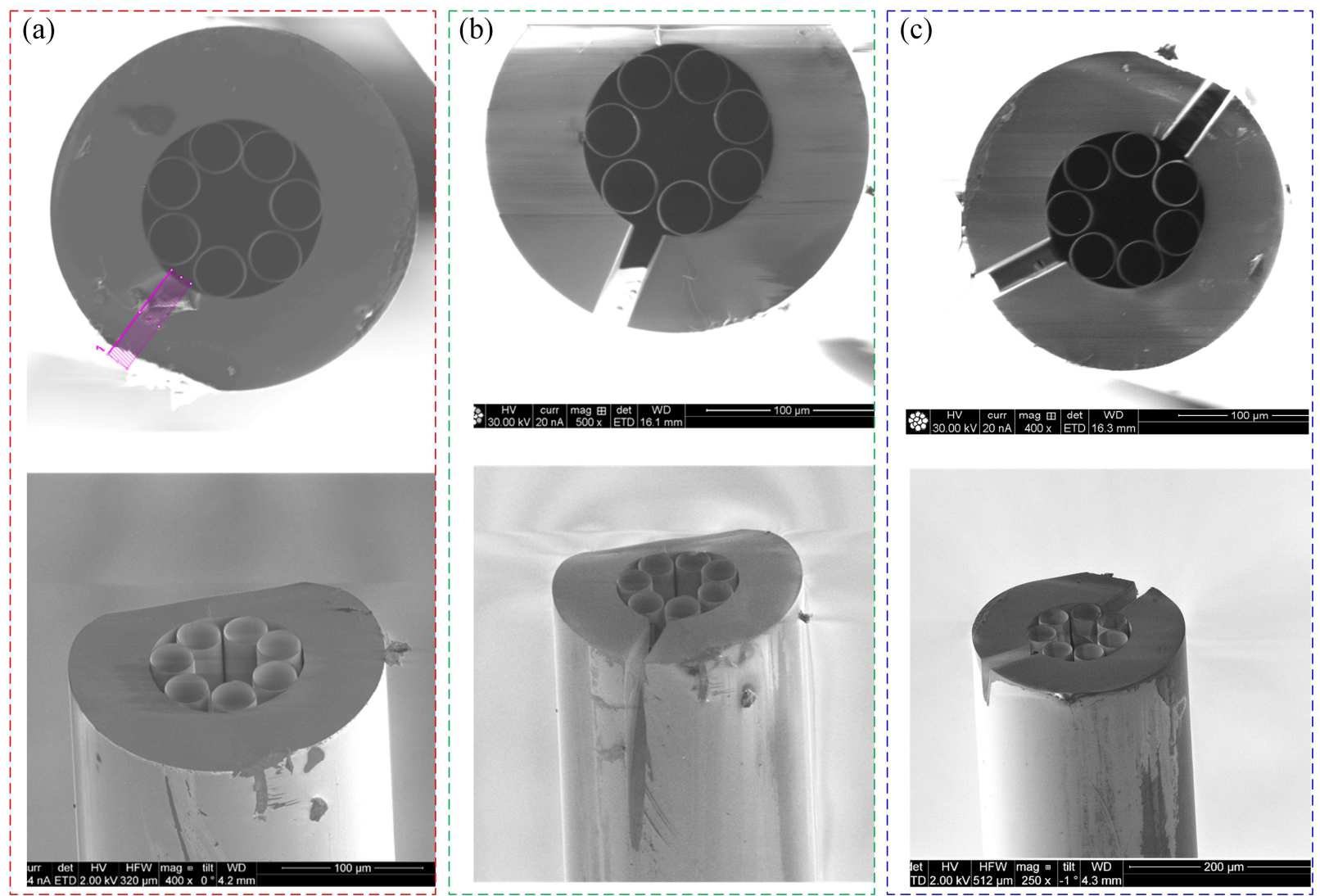

Figure 2. SEM images of ARHCF (a) to be drilled, (b) one milled channel, (c) two milled channels. The top row shows the fiber facet images, while the bottom row shows the lateral images. The non-symmetry of fiber facets in lateral images is an artefact due to angle-correction in SEM image reconstruction, details of this can be found in Ref. ${ }^{28}$. 
The ARHCF is fabricated by following the designed parameters in Figure 1(a). The stack and draw technique was used for fabrication: seven silica tubes are stacked in a larger silica tube with a broader inner diameter, forming the final preform. The preform is put in the furnace and heated above the glass transition temperature of silica, under controlled pressures in core and inner capillary regions, to prevent hole-collapsing. The detailed fabrication process is referred to Ref. ${ }^{29}$. On the other hand, FIB is an advanced and versatile technique for milling and sputtering of materials. It allows direct patterning on a sample through the bombardment of ions on the sample. Unlike laser ablation, the area of interest is predefined, as shown in Figure 2(a) and accurately controlled throughout the milling process. The technique involves dual-beam instruments containing an SEM imaging system, and an FIB gun that emits highly energetic ions, such as gallium ion $\left(\mathrm{Ga}^{+}\right)$beam. $\mathrm{Ga}^{+}$is preferred because it has a low melting point and vapor pressure, which simplifies operations ${ }^{30}$. The main advantage of FIB is its high alignment precision, in that it can mill spot sizes less than $10 \mathrm{~nm}^{31}$. Similar to scanning electron microscopy, in FIB, highly energetic electrons can accumulate on insulating surfaces. Therefore, coating of fiber with a conductive materials is necessary to reduce surface charging and allow high magnification levels. These electron charges can cause random deflection of the incident ion beam and thus damage the milling profile.

The sample was prepared by stripping the polymer coating from the fiber tip and cleaving the fiber with tension cleaver to get a flat end facet. To minimize the charging effect, the fiber is coated with a $5 \mathrm{~nm}$ thick layer of gold using sputter coater (Cressington, model 208HR, targets: $\mathrm{Au} / \mathrm{Cr}$ ). Moreover, the fiber is fixed on the top of conductive carbon tape attached to a grounded sample holder. Additional carbon tape is used to fix the fiber in place. The fiber is then placed in the FIB system (FEI Quanta 200 3D SEM-FIB), with the facet placed at a $\sim 90^{\circ}$ incident angle between the surface normal and the ion beam. This significantly increases the milling rate, since the FIB sputtering yield roughly increases with $1 / \cos (\theta){ }^{32,33}$, where $\theta$ here is the angle between the fiber-facet normal and direction of the FIB gun. The milling procedure is performed with an acceleration voltage of $30 \mathrm{kV}$ and current of $20 \mathrm{nA}$. This high ion current leads to a high milling rate, a channel of $50 \mu \mathrm{m}$ depth by taking $\sim 30$ minutes.

The drilled micro-channels are presented in Fig. 2(b) and 2(c), with a $43 \mu \mathrm{m}$ x $30 \mu \mathrm{m}$ diameter, and a $\sim 50 \mu \mathrm{m}$ depth through the outer silica cladding part. It can be seen that they are precisely adjacent to the gap between two capillaries, therefore maintaining the completeness of the capillaries' structure and consequently minimizing the induced extra loss.

\section{CONCLUSION}

In this work, we explored the utilization of FIB milling on nodeless ARHCF and we demonstrated that how micro-channels can be achieved on the fiber facets without damaging the cladding capillaries, thus minimizing the extra loss induced by the micromachining. This design enables access for gas/liquid to the cores when the fiber facets are spliced with solid core fibers. Since the important applications of HCF with low loss channels are obvious and have already been demonstrated

in several papers ${ }^{4,6,33-37}$, here we focus on the fabrication approach rather than specific sensing applications. The presented work is an additional step towards achieving an all-fiberized, low-loss fiber fluid-cell for optofluidic applications.

\section{ACKNOWLEDGEMENTS}

This work is supported by the Danmarks Frie Forskningsfond Hi-SPEC project (Grant No. 8022-00091B), Multi-BRAIN project funded by the Lundbeck Foundation (R276-2018-869), ECOMETA (Grant No. 6150-00030B) and US ARO (Grant No. W911NF-19-1-0426). Abubakar I. Adamu is now with Lumenisity Ltd, United Kingdom.

\section{REFERENCES}

[1] Markos, C., Travers, J. C., Abdolvand, A., Eggleton, B. J. and Bang, O., "Hybrid photonic-crystal fiber," Rev. Mod. Phys. 89(4), 045003 (2017).

[2] Cregan, R. F., Mangan, B. J., Knight, J. C., Birks, T. A., Russell, P. S. J., Roberts, P. J. and Allan, D. C., "Singlemode photonic band gap guidance of light in air," Science 285(5433), 1537 - 1539 (1999).

[3] Markos, C., Antonopoulos, G. and Kakarantzas, G., "Broadband Guidance in a Hollow-Core Photonic Crystal Fiber With Polymer-Filled Cladding," IEEE Photonics Technol. Lett. 25(20), 2003-2006 (2013).

[4] Hensley, C. J., Broaddus, D. H., Schaffer, C. B. and Gaeta, A. L., "Photonic band-gap fiber gas cell fabricated using femtosecond micromachining," Opt. Express 15(11), 6690-6695 (2007). 
[5] Jin, W., Xuan, H. F. and Ho, H. L., "Sensing with hollow-core photonic bandgap fibers," Meas. Sci. Technol. 21(9), 94014 (2010).

[6] Van Brakel, A., Grivas, C., Petrovich, M. N. and Richardson, D. J., "Micro-channels machined in microstructured optical fibers by femtosecond laser," Opt. Express 15(14), 8731-8736 (2007).

[7] Couny, F., Benabid, F. and Light, P. S., "Reduction of fresnel back-reflection at splice interface between hollow core PCF and single-mode fiber," IEEE Photonics Technol. Lett. 19(13), 1020-1022 (2007).

[8] Wynne, R. M., Barabadi, B., Creedon, K. J. and Ortega, A., "Sub-minute response time of a hollow-core photonic bandgap fiber gas sensor," J. Light. Technol. 27(11), 1590-1596 (2009).

[9] Adamu, A. I., Ozturk, F. E. and Bayindir, M., "Binary coded identification of industrial chemical vapors with an optofluidic nose," Appl. Opt. 55(36), 10247-10254 (2016).

[10] Cox, F. M., Argyros, A. and Large, M. C. J., "Liquid-filled hollow core microstructured polymer optical fiber," Opt. Express 14(9), 4135-4140 (2006).

[11] Qian, W., Zhao, C.-L., Wang, Y., Chan, C. C., Liu, S. and Jin, W., "Partially liquid-filled hollow-core photonic crystal fiber polarizer," Opt. Lett. 36(16), 3296-3298 (2011).

[12] Zeltner, R., Bykov, D. S., Xie, S., Euser, T. G. and Russell, P. S. J., "Fluorescence-based remote irradiation sensor in liquid-filled hollow-core photonic crystal fiber," Appl. Phys. Lett. 108(23), 231107 (2016).

[13] Adamu, A. I., Habib, M. S., Petersen, C. R., Lopez, J. E. A., Zhou, B., Schülzgen, A., Bache, M., Amezcua-Correa, R., Bang, O. and Markos, C., "Deep-UV to mid-IR supercontinuum generation driven by mid-IR ultrashort pulses in a gas-filled hollow-core fiber," Sci. Rep. 9(1), 4446 (2019).

[14] Wang, Y., Dasa, M. K., Adamu, A. I., Antonio-Lopez, J. E., Habib, M. S., Amezcua-Correa, R., Bang, O. and Markos, C., "High pulse energy and quantum efficiency mid-infrared gas Raman fiber laser targeting CO2 absorption at $4.2 \mu \mathrm{m}$," Opt. Lett. 45(7), 1938-1941 (2020).

[15] Adamu, A. I., Wang, Y., Habib, M. S., Dasa, M. K., Antonio-Lopez, J. E., Amezcua-Correa, R., Bang, O. and Markos, C., "Multi-wavelength high-energy gas-filled fiber Raman laser spanning from $1.53 \mu \mathrm{m}$ to $2.4 \mu \mathrm{m}$," Opt. Lett. 46(3), 452-455 (2021).

[16] Cordeiro, C. M. B., dos Santos, E. M., Brito Cruz, C. H., de Matos, C. J. S. and Ferreira, D. S., "Lateral access to the holes of photonic crystal fibers - selective filling and sensing applications," Opt. Express 14(18), 8403-8412 (2006).

[17] Fokoua, E. N., Richardson, D. J. and Poletti, F., "Impact of structural distortions on the performance of hollowcore photonic bandgap fibers," Opt. Express 22(3), 2735-2744 (2014).

[18] Sakr, H., Chen, Y., Bradley, T. D., Jasion, G. T., Hayes, J. R., Davidson, I. A., Fokoua, E. N., Wheeler, N. V, Richardson, D. J. and Poletti, F., "Advances in hollow core fiber for the $1 \mu \mathrm{m}$ and visible wavelength regions," OSA Adv. Photonics Congr., SoW1H.5 (2020).

[19] Yu, F., Wadsworth, W. J. and Knight, J. C., "Low loss silica hollow core fibers for 3-4 $\mu \mathrm{m}$ spectral region," Opt. Express 20(10), 11153-11158 (2012).

[20] Poletti, F., Hayes, J. R. and Richardson, D. J., "Optimising the performances of hollow antiresonant fibres," 37th Eur. Conf. Expo. Opt. Commun., Mo.2.LeCervin.2 (2011).

[21] Poletti, F., "Nested antiresonant nodeless hollow core fiber," Opt. Express 22(20), 23807-23828 (2014).

[22] Kolyadin, A. N., Kosolapov, A. F., Pryamikov, A. D., Biriukov, A. S., Plotnichenko, V. G. and Dianov, E. M., "Light transmission in negative curvature hollow core fiber in extremely high material loss region," Opt. Express 21(8), 9514-9519 (2013).

[23] Min, Y., Filipkowski, A., Stępniewski, G., Klimczak, M., Zhao, L., Buczyński, R., "Fusion splicing and termination of silica hollow core anti-resonant fibers with single mode fibers," Conference programme, 88 (2020).

[24] Thapa, R., Knabe, K., Corwin, K. L. and Washburn, B. R., "Arc fusion splicing of hollow-core photonic bandgap fibers for gas-filled fiber cells," Opt. Express 14(21), 9576-9583 (2006).

[25] Hou, M., Zhu, F., Wang, Y., Wang, Y., Liao, C., Liu, S. and Lu, P., "Antiresonant reflecting guidance mechanism in hollow-core fiber for gas pressure sensing," Opt. Express 24(24), 27890-27898 (2016).

[26] Novo, C. C., Choudhury, D., Siwicki, B., Thomson, R. R. and Shephard, J. D., "Femtosecond laser machining of hollow-core negative curvature fibres," Opt. Express 28(17), 25491-25501 (2020).

[27] Song, P., Phoong, K. Y. and Bird, D., "Quantitative analysis of anti-resonance in single-ring, hollow-core fibres," Opt. Express 27(20), 27745-27760 (2019).

[28] JIN, P. and LI, X., "Correction of image drift and distortion in a scanning electron microscopy," J. Microsc. 260(3), 268-280 (2015).

[29] Antonio-Lopez, J. E., Habib, S., Newkirk, A. Van, Lopez-Galmiche, G., Eznaveh, Z. S., Alvarado-Zacarias, J. C., 
Bang, O., Bache, M., Schülzgen, A. and Correa, R. A., "Antiresonant hollow core fiber with seven nested capillaries,” 2016 IEEE Photonics Conf., 402-403 (2016).

[30] Fu, Y., "Integration of microdiffractive lens with continuous relief with vertical-cavity surface-emitting lasers using focused ion beam direct milling," IEEE Photonics Technol. Lett. 13(5), 424-426 (2001).

[31] Nguyen, N.-T., "Micromixers: fundamentals, design and fabrication," William Andrew, 1-328 (2011).

[32] Reyntjens, S. and Puers, R., "A review of focused ion beam applications in microsystem technology," J. Micromechanics Microengineering 11(4), 287-300 (2001).

[33] Yuan, W., Wang, F., Savenko, A., Petersen, D. H. and Bang, O., "Note: Optical fiber milled by focused ion beam and its application for Fabry-Pérot refractive index sensor," Rev. Sci. Instrum. 82(7), 76103 (2011).

[34] Adamu, A. I., Dasa, M. K., Bang, O. and Markos, C., "Multispecies continuous gas detection with supercontinuum laser at telecommunication wavelength," IEEE Sens. J. 20(18), 10591-10597 (2020).

[35] Nikodem, M., Gomółka, G., Klimczak, M., Pysz, D. and Buczyński, R., "Demonstration of mid-infrared gas sensing using an anti-resonant hollow core fiber and a quantum cascade laser," Opt. Express 27(25), 36350-36357 (2019).

[36] Litchinitser, N. M. and Poliakov, E., "Antiresonant guiding microstructured optical fibers for sensing applications," Appl. Phys. B 81(2), 347-351 (2005).

[37] Gao, H., Jiang, Y., Zhang, L., Cui, Y., Jiang, Y., Jia, J. and Jiang, L., “Antiresonant mechanism based selftemperature-calibrated fiber optic Fabry-Perot gas pressure sensors," Opt. Express 27(16), 22181-22189 (2019). 